\title{
Effect of Dexmedetomidine on Intestinal Barrier in Patients Undergoing Gastrointestinal Surgery - A Single-Centre Randomized Clinical Trial
}

\section{Yupeng Qi}

The First Affiliated Hospital of Wannan Medical College: Yijishan Hospital of Wannan Medical College

\section{Wenjing Ma}

The First Affiliated Hospital of Wannan Medical College: Yijishan Hospital of Wannan Medical College

\section{Yingya Cao}

The First Affiliated Hospital of Wannan Medical College: Yijishan Hospital of Wannan Medical College

\section{Qun Chen}

The First Affiliated Hospital of Wannan Medical College: Yijishan Hospital of Wannan Medical College

\section{Qiancheng Xu}

The First Affiliated Hospital of Wannan Medical College: Yijishan Hospital of Wannan Medical College

Shi Xiao

The First Affiliated Hospital of Wannan Medical College: Yijishan Hospital of Wannan Medical College

\section{Weihua Lu}

The First Affiliated Hospital of Wannan Medical College: Yijishan Hospital of Wannan Medical College zhen wang ( $\nabla$ zhenwang80@126.com )

First Affiliated Hospital of Wannan Medical College https://orcid.org/0000-0002-8051-5471

\section{Research}

Keywords: Gastrointestinal Surgery, Intestinal Barrier, Dexmedetomidine, Cholinergic Anti-inflammatory Pathway (CAP)

Posted Date: January 21st, 2021

DOl: https://doi.org/10.21203/rs.3.rs-150967/v1

License: (c) (i) This work is licensed under a Creative Commons Attribution 4.0 International License. Read Full License 


\section{Abstract}

Background: Gastrointestinal failure accounts for death in critically ill patients. This study aimed to explore the effect and mechanism of dexmedetomidine (DEX) in intestinal barrier function in critically ill patients undergoing gastrointestinal surgery.

Methods: Patients undergoing gastrointestinal surgery were randomized into a DEX group $(n=21)$ or an MID group ( $n=21)$. Sufentanil was used in both groups for analgesia. In the DEX group, DEX was loaded $(1 \mu \mathrm{g} / \mathrm{kg})$ before sedation and was infused $(0.7 \mu \mathrm{g} / \mathrm{kg} / \mathrm{h})$ during sedation. The mean arterial pressure (MAP), heart rate (HR), borborygmus resumption time (BRT), first defecation time (FDT), stay of ICU and hospital were observed. The DAO, D-LAC, TNF-a, IL-6 and $a 7 n A C h R$ levels in plasma or haemocytes were detected before the start of the sedation $(0 \mathrm{~h})$ and after the sedation $(24 \mathrm{~h})$.

Results: There were no significant differences in age, sex, BMI, APACHE II score, SOFA $(P>0.05)$. The MAP between 0 and $24 \mathrm{~h}$ presented no significant difference between the groups $(P>0.05)$, but HR was significantly slower in the DEX group $(P=0.042)$. The recovery time of bowel sounds was significantly earlier in the DEX group $(P=0.034)$. Both of the stay of ICU $(P=0.016)$ and hospital $(P=0.031)$ were significantly shorter in the DEX group. The expression of a7nAChR in the DEX group was significantly higher at $24 \mathrm{~h}$ than at $0 \mathrm{~h}(\mathrm{P}=0.002)$. The $\mathrm{D}$-LAC decreased significantly in the DEX group than MID group at $24 \mathrm{~h}(P=0.016)$.

Conclusions: DEX maintained the integrity of the intestinal barrier in patients undergoing gastrointestinal surgery through the cholinergic anti-inflammatory pathway.

Trial registration:ChiCTR1900024367. Registered 7 July 2019-Retrospectively registered, http://www.chictr.org.cn/showproj.aspx?proj=40832

\section{Introduction}

The gastrointestinal tract provides the largest storage of bacterial organisms and endotoxins,and also the most sensitive to ischaemia and hypoperfusion of the human body. Intestinal epithelial tissue can resist the invasion of pathogens, produce and secrete antimicrobial peptides, and maintain homeostasis[1, 2]. Because of mechanical injury, intestinal surgery can lead to Intestinal barrier function dysfunction[3]. A large number stress factors can destroy the intestinal barrier, disturb the intestinal flora's homeostasis, disrupt immune function, and release intestinal bacteria. At the same time, the bacterial metabolites enter the blood, causing gut origin sepsis (GOS)[4, 5].

The gastrointestinal tract is the largest organ innervated by nerve fibres. The inflammatory reflex formed by the vagus nerve could play an important role in intestinal immune regulation. Studies[6, 7] have shown that upregulation of vagal nerve activity could improve the intestinal permeability barrier. Dexmedetomidine (DEX) can improve the activity of the vagus nerve and activate the expression of a7nAChR[8-10]. Studies[11-17] have shown that DEX has a definite anti-inflammatory effect, and this 
effect is similar to that of direct electrical stimulation of the vagus nerve, but whether it can further improve intestinal permeability in patients with gastrointestinal surgery is unclear[18]. Therefore, our study was conducted to investigate whether DEX could improve intestinal permeability in intestinal surgery and to explore its possible mechanism.

\section{Methods}

\subsection{Study subjects}

This randomized,double-blinded $\square$ prospective $\llbracket$ controlled study was performed in accordance with the Declaration of Helsinki, approved by the Institutional Review Board of the First Affiliated Hospital of Wannan Medical College (approval number: WAN 2015-18) and registered with the Chinese Clinical Trial Registry at www.chictr.org(registration number: ChiCTR1900024367).

Patients were included according to the following criteria: 1) patients undergoing gastrointestinal surgery; 2) patients who received ventilator-assisted ventilation intubated through the oral trachea for $12 \mathrm{~h}$ and an estimated ventilation time of more than $24 h ; 3$ ) patients who needed analgesia and sedation; and 4) an informed consent form signed by the patient or family member. Patients who met any of the following criteria were excluded: 1) women who were pregnant or lactating; 2) patients younger than 18 years old; 3) patients with heart rates (HRs) lower than $55 \mathrm{bpm}$; 4) patients with a high atrioventricular block without a cardiac pacemaker; 5) patients diagnosed with acute liver failure; 6) patients diagnosed with cerebrovascular accident; and 7) patients diagnosed with dementia.

Using a computer-generated random number table, patients were randomly assigned to the DEX group or the MID group (control group). DEX was loaded $(1 \mathrm{mg} / \mathrm{kg}$ ) before sedation and was infused ( 0.3 $\mathrm{mg} / \mathrm{kg} / \mathrm{h}$ ) during sedation. Analgesia and sedation were performed according to the following procedure: (the RASS score was maintained at -2 to 1) [19] (Figure 1).

\subsection{Sample size}

Fifty patients who provided written consent were enrolled between June 2017 and May 2019.

\subsection{Procedure}

To eliminate any possible effects of surgical technique, all of the procedures were performed by the same surgical group. To maintain blinding, the clinicians who prepared and performed the sedation and analgesia were not involved in management or assessments unless an emergency occurred. The investigators and patients were blinded to the intervention.

The patients of gender, age, weight, diagnosis, APACHE II score, SOFA score, hourly heart rate and blood pressure, recovery time of bowel sounds, first defecation time, length of ICU hospital stay and length of hospital stay were recorded. 
The patients' sex, age, weight, diagnosis, APACHE II score, SOFA score, hourly HR and blood pressure, recovery time of bowel sounds, first defecation time, length of ICU hospital stay and length of hospital stay were recorded.

Blood was collected $0 \mathrm{~h}$ and $24 \mathrm{~h}$. The blood was centrifuged at $3000 \mathrm{rpm}$ for $15 \mathrm{~min}$. The supernatant was carefully absorbed into a 2-ml EP tube with a transfusion gun (avoiding absorbing blood cells) to detect TNF-a, D-Lac, and IL-6. The contents of D-Lac, DAO, TNF-a and IL-6 in blood were detected strictly using ELISA kit instructions. Blood cells were collected to detect the changes in a7nAChR mRNA using real-time PCR (total RNA extraction, total RNA reverse transcription into CDNA, and quantitative analysis of a7nAChR mRNA, with $\beta$-actin as an internal reference).

\subsection{The outcomes}

The primary outcomes included the DAO, D-LAC, and a7nAChR levels in plasma or haemocytes.

The secondary outcomes included the mean arterial pressure (MAP), HR, borborygmus resumption time (BRT), first defecation time (FDT), length of ICU hospital stay and length of hospital stay.

\subsection{Statistical analysis}

SPSS software, version 22.0 (Chicago, IL, USA), was used for data analysis. All of the data were tested by normal distribution and homogeneity of variance. The measurement data with normal distribution are expressed as the mean \pm standard deviation $(x \pm s)$. The Mann-Whitney U nonparametric test was used to compare the data and rates of non-normal distribution. $\mathrm{P}<0.05$ was considered statistically significant.

\section{Results}

A total of 50 patients were included in this study. Four patients refused to be followed up in the middle of the study. Four patients were excluded because of incomplete follow-up data. Finally, the remaining 42 patients were included in the statistical analysis(Figure 2).

\subsection{Demographic and clinical characteristics of the DEX and MID groups}

There were no significant differences in age, sex, BMI, APACHE II score, SOFA score or RASS score between the DEX and MID groups $(P>0.05)$.

There was no significant difference in blood pressure between the two groups $(P>0.05)$, but the HR in the DEX group was significantly lower than that in the MID group $(P=0.042)$. The recovery time of bowel sounds in the DEX group was significantly shorter than that in the MID group $(P=0.034)$, and there was no significant difference in the first defecation time between the two groups $(P>0.05)$. Compared with the MID group, the DEX group had a significantly shorter length of ICU hospital stay $(P=0.016)$ and length of hospital stay $(P=0.031)$. (Table 1$)$. 
Table 1

Demographic and clinical characteristics of the DEX and MID groups

\begin{tabular}{|c|c|c|c|c|}
\hline & $\begin{array}{l}\text { DEX Group } \\
(\mathrm{n}=\mathbf{2 1})\end{array}$ & $\begin{array}{l}\text { MID Group } \\
(n=21)\end{array}$ & $\mathbf{F}$ & $\mathbf{P}$ \\
\hline Age(years) & $69.19 \pm 8.52$ & $69.38 \pm 9.18$ & 0.242 & 0.945 \\
\hline Sex (male/female) & $16 / 5$ & $16 / 5$ & - & - \\
\hline $\operatorname{BMI}\left(\mathrm{kg} / \mathrm{m}^{2}\right)$ & $20.40 \pm 2.77$ & $21.70 \pm 3.60$ & 0.627 & 0.196 \\
\hline APACHE II score & $15.86 \pm 3.62$ & $15.67 \pm 4.07$ & 0.712 & 0.323 \\
\hline SOFA score & $9.15 \pm 2.35$ & $9.25 \pm 3.02$ & 2.500 & 0.91 \\
\hline RASS score & $-0.33 \pm 1.11$ & $-0.48 \pm 1.08$ & 0.003 & 0.675 \\
\hline MAP (mm Hg) & $87.20 \pm 9.70$ & $87.98 \pm 9.66$ & 0.458 & 0.795 \\
\hline $\mathrm{HR}(\mathrm{bpm})$ & $72.63 \pm 8.21$ & $81.51 \pm 17.31$ & 6.867 & 0.042 \\
\hline BRT(d) & $2.95 \pm 0.97$ & $3.76 \pm 1.37$ & 7.290 & 0.034 \\
\hline $\mathrm{FDT}(\mathrm{d})$ & $6.24 \pm 2.10$ & $7.38 \pm 2.00$ & 0.000 & 0.077 \\
\hline Length of hospital stay(d) & $18.86 \pm 8.12$ & $24.74 \pm 8.91$ & 0.350 & 0.031 \\
\hline Length of ICU stay (d) & $2.76 \pm 0.77$ & $3.71 \pm 1.56$ & 3.910 & 0.016 \\
\hline \multicolumn{5}{|c|}{$\begin{array}{l}\text { BRT: borborygmus resumption time, FDT: first defecation time, BMI: body mass index, APACHE II: } \\
\text { Acute Physiology and Chronic Health Evaluation, SOFA: Sequential Organ Failure Assessment, RASS } \\
\text { Richmond Agitation and Sedation Scale, MAP: mean arterial pressure HR: heart rate, Length of } \\
\text { hospital stay: duration of postoperative hospitalization stay, Length of ICU stay: duration of ICU } \\
\text { hospitalization stay }\end{array}$} \\
\hline
\end{tabular}

\subsection{Changes in indicators of intestinal inflammation and permeability in the DEX and MID groups}

TNF-a decreased significantly in the DEX group at $0 \mathrm{~h}$ compared with $24 \mathrm{~h}$, but IL- 6 levels did not change significantly in the DEX group. TNF-a and IL- 6 levels did not change significantly in the MID group at $0 \mathrm{~h}$ compared with $24 \mathrm{~h}$. There was no significant difference in TNF-a and IL-6 levels between the DEX and MID groups at $0 \mathrm{~h}(P>0.05)$. Compared with the MID group, the DEX group decreased the production of TNF- $a(P=0.044)$ at $24 \mathrm{~h}$, but there was no significant difference in IL- 6 between the two groups.

The serum levels of DAO and D-LAC in the DEX group were significantly different at $0 \mathrm{~h}$ than at $24 \mathrm{~h}(\mathrm{P}<$ $0.05)$, but there was no significant difference between the MID group at $0 \mathrm{~h}$ and at $24 \mathrm{~h}(\mathrm{P}>0.05)$, and the serum levels of DAO and D-LAC in the DEX group showed no significant differences compared with those in the MID group at $0 \mathrm{~h}(\mathrm{P}>0.05)$, while DEX reduced the production of $\mathrm{D}$-LAC more than MID at $0 \mathrm{~h}$ than at $24 \mathrm{~h}(\mathrm{P}<0.05)($ Table 2$)$. 
Table 2

Changes in the indicators of intestinal inflammation and permeability in the DEX and MID groups

\begin{tabular}{|c|c|c|c|c|c|}
\hline & & DEX Group & MID Group & $F$ & $\mathbf{P}$ \\
\hline IL-6 & $\mathrm{Oh}$ & $69.18 \pm 19.14$ & $64.23 \pm 10.76$ & 8.774 & 0.312 \\
\hline$(\mathrm{ng} / \mathrm{l})$ & $24 \mathrm{~h}$ & $67.97 \pm 18.28$ & $64.21 \pm 13.34$ & 7.767 & 0.429 \\
\hline TNF-a & $\mathrm{Oh}$ & $124.61 \pm 36.98$ & $105.52 \pm 28.14$ & 2.499 & 0.067 \\
\hline$(\mathrm{ng} / \mathrm{l})$ & $24 \mathrm{~h}$ & $99.03 \pm 30.49^{\square}$ & $116.24 \pm 22.67$ & 1.106 & 0.044 \\
\hline D-LAC & $\mathrm{Oh}$ & $40.35 \pm 8.00$ & $40.22 \pm 8.23$ & 0.003 & 0.960 \\
\hline$(\mu \mathrm{mol} / \mathrm{ml})$ & $24 \mathrm{~h}$ & $34.00 \pm 5.68^{\square}$ & $39.13 \pm 7.39$ & 0.928 & 0.016 \\
\hline DAO & $\mathrm{Oh}$ & $72.91 \pm 14.54$ & $67.15 \pm 17.70$ & 0.034 & 0.209 \\
\hline$(\mathrm{ng} / \mathrm{l})$ & $24 \mathrm{~h}$ & $63.06 \pm 15.08^{\square}$ & $63.06 \pm 15.08$ & 6.359 & 0.072 \\
\hline \multicolumn{6}{|c|}{ Note: * Represents an intra-group comparison $(P<0.05)$} \\
\hline \multicolumn{6}{|c|}{ IL-6: interleukin-6; TNF-a: tumour necrosis factor alpha; D-LAC: D-lactate; DAO: diamine oxidas } \\
\hline
\end{tabular}

\subsection{Comparison of a7nAChR levels between the two groups at $0 \mathrm{~h}$ and at $24 \mathrm{~h}$}

The level of a7nAChR in the DEX group was significantly higher than that in the MID group $(P<0.05)$, but there was no significant change in the level of $a 7 n A C h R$ in the MID group between $0 \mathrm{~h}$ and $24 \mathrm{~h}(P>0.05)$. There was no significant difference in the level of a7nAChR between the DEX and MID groups at $0 \mathrm{~h}$, and the level of a7nAChR in the DEX group was significantly higher than that in the MID group at $24 \mathrm{~h}(\mathrm{P}=$ 0.015) (Table 3).

Table 3

Changes in a7nAChR levels before and after treatment in the two groups

\begin{tabular}{|lllll|}
\hline & \multicolumn{2}{c}{ DEX Group } & MID Group & $P$ \\
\hline a7nAChR & $\mathrm{Oh}$ & $0.25(0.18-0.63)$ & $0.52(0.15-0.92)$ & 0.308 \\
\cline { 2 - 5 } & $24 \mathrm{~h}$ & $0.62(0.43-1.20)$ & $0.22(0.10-0.79)$ & 0.015 \\
\hline \multicolumn{4}{|l}{ Note: * Represents an intragroup comparison $(\mathrm{P}<0.05)$. a7nAChR: a7-nicotinic acetylcholine receptor } \\
\hline
\end{tabular}

\section{Discussion}

The gastrointestinal mucosa is very sensitive, and gastrointestinal surgery always destroy the intestinal mechanical barrier. At the same time, the gastrointestinal muscle layer is filled with macrophages, many of which are released when stimulated,further promoting the release of cell factors, prostaglandins and 
other factors. Therefore, when mucosal injuries occur, they can cause local and systemic inflammatory responses and even sepsis, resulting in postoperative gastrointestinal dysfunction[20].

In recent years, an increasing number of scholars have proposed that the intestinal tract plays an important role in the development of sepsis. Some scholars[21] have proposed that the intestinal tract is not only the first organ involved in sepsis but also the "initiating organ" of sepsis. Approximately $30 \%$ of sepsis patients who die of multiple organ dysfunction syndrome (MODS) do not clinically exhibit a primary infection focus, but bacteria similar to intestinal bacteria can be found in their blood cultures[2225].

The intestinal tract is often involved earlier and recovers later in the disease course. The study found that abdominal distension, intra-abdominal pressure and the time to recovery of intestinal function were significantly delayed in patients with sepsis. The mechanism of injury might be that the gastrointestinal mucosa and villi are rich in blood flow, sensitive to ischaemia and hypoxia, and vulnerable to damage under hypoperfusion. When sepsis occurs, the circulatory blood volume decreases, and the intestinal blood flow decreases significantly. When the systemic circulatory blood volume decreases by $10 \%$, gastrointestinal blood perfusion decreases by nearly half. Long-term hypoperfusion can cause intestinal mucosal cell oedema, villous degeneration and necrosis, damage or even loss of tight junctions between cells, and increased intestinal permeability. If not controlled in time, this condition will eventually result in MODS $[1,2,21]$. Therefore, controlling inflammatory reactions, maintaining intestinal function, protecting the intestinal barrier integrity and avoiding intestinal bacteria and endotoxin release into the blood are the key points for preventing MODS[26].

Recent studies have found that the "cholinergic anti-inflammatory pathway" (CAP) is a neuroimmune regulatory pathway with obvious anti-inflammatory effects[27]. The CAP is composed mainly of the

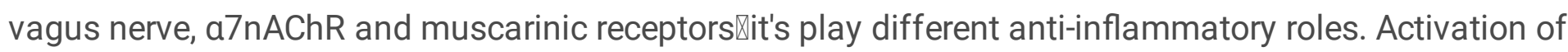
the CAP can effectively reduce the release of TNF- $\alpha$, IL-6, IL-1 $\beta$ and other inflammatory factors and can significantly inhibit the inflammatory reaction caused by various local and systemic causes[28, 29]. $\mathrm{a} 7 \mathrm{nAChR}$ is an important target in the CAP, and it has been a popular research topic in recent years.

Compared with other nicotinic acetylcholine receptors, $\mathrm{a} 7 \mathrm{nAChR}$ can be activated rapidly by agonists. The permeability of these receptors to calcium ions increases when activated and can allow for the inflow of calcium ions without causing the depolarization of cell membranes. Therefore, a7nAChR can participate in the regulation of calcium-related events conveniently and quickly. Research have shown that the CAP mainly plays a role through a7nAchR[30]. Directional knockout of the a7nAChR gene or vagotomy can reduce the activity of the CAP and aggravate the inflammatory reaction in various inflammatory model animals. A preliminary study based on clinical sepsis patients showed that high expression of a7nAChR in peripheral blood mononuclear cells was associated with reduced inflammatory status and improved prognosis[31].

DEX is a new type of a2-adrenergic receptor agonist that can simultaneously act on the central and peripheral nervous systems, regulate autonomic nervous activity, and produce dose-dependent sedative, 
hypnotic and anti-anxiety effects. At the same time, DEX can reduce the incidence of delirium in patients, and it was widely used in the sedative treatment of critically ill patients [26, 32-34]. Our study found that, compared to MID, DEX had no significant effect on blood pressure at the depth of shallow sedation, At the same time, DEX can also shorten the length of ICU stay.

Several animal experiments on acute inflammation have shown that DEX significantly inhibits the overrelease of TNF- $a, \mathrm{IL}-1 \beta, \mathrm{IL}-6$ and other inflammatory factors. Clinical studies have also found that DEX use in general anaesthesia can significantly reduce the levels of plasma inflammatory factors in perioperative patients. Especially in patients with severe sepsis caused by intestinal obstruction, DEX treatment can not only reduce the release of inflammatory factors but also reduce the increased intraabdominal pressure of sepsis patients[14, 15]. Our study found that the borborygmus resumption time (BRT) in the DEX group was significantly shorter than that in the control group. At the same time, our study found that the level of TNF-a in the DEX group was significantly lower, showing that the clinical sedative dose of DEX could produce an obvious anti-inflammatory effect and block the cascade reaction of inflammation.

Many studies have shown that DEX exerts its anti-inflammatory effects by activating the CAP of the vagus nerve and the $\mathrm{a}-2$ adrenergic receptor, and its activation of the CAP is achieved mainly by activating a7nAChR.[35]. In our study, we found that DEX increased the expression of a7nAChR in peripheral blood mononuclear cells.

When the gut was damaged, IFN- $y$, TNF- $\alpha$ and IL-1 $\beta$ inflammatory factors, through the CAP, activate myosin light streptokinase within intestinal epithelial cells, resulting in myosin light chain phosphorylation, promoting a variety of proteins (such as adhesion molecule- 1 , occludin, and claudin) in the closely connected intestinal epithelial cells, thereby inducing functional changes and eventually damaging the integrity of tight junctions[36,37]. Therefore, the key to maintaining intestinal barrier function is inhibiting the inflammatory response. The serum D-Lac level are often used as important reference indicators for evaluating intestinal barrier function[38]. Our study showed that the level of D-Lac decreased significantly in the DEX group, indicating that DEX could significantly improve intestinal permeability in patients undergoing gastrointestinal surgery. At the same time, combined with the increased expression of a7nAChR in peripheral blood mononuclear cells, it can be inferred that mechanism might be related to the activation of a7nAChR, increased CAP activity and inhibition of the intestinal inflammatory response

\section{Conclusions}

DEX is more suitable to patients undergoing gastrointestinal surgery. as a sedative, due to the protection of intestinal barrier. The mechanism might be related to the activation of a7nAChR, increased CAP activity and inhibition of the intestinal inflammatory response.

\section{Declarations}


Authors' contributions

WHL and ZW conceived and designed the study. YPQ,YYC and WJM performed the experiments and wrote the paper.XS,QC and YYC contributed essential materials, and YPQ and YYC analysed and interpreted the data. All of the authors read and approved the final manuscript.

\section{Funding}

The Key Laboratory of Noncoding RNA Transformation Research of Anhui Higher Education Institution (RNA201910).

Anhui Province Natural Science Foundation for Youth (1908085QH360)

Funding of "Peak" Training Program for Scientific Research of Yijishan Hospital, Wannan Medical College (GF2019G08)

\section{Availability of data and materials}

The datasets generated during the current study are available from the corresponding author on reasonable request.

\section{Ethics approval and consent to participate}

The study was approved by the local ethics committee (Ethics Committee of the First Affiliated Hospital of Wannan Medical College, protocol number 201518, date of approval 18 November 2015).

\section{Consent for publication}

Not applicable

\section{Competing interests}

The authors declare that they have no competing interests.

\section{Acknowledgements}

Grateful acknowledgement is made to my supervisors Mr. Weihua Lu and Mr. Zhen Wang, who gave me considerable help by means of suggestion, comments and criticism. Their encouragement and unwavering support have sustained me through frustration and depression. Without their pushing me ahead, the completion of this thesis would be impossible. In addition, I deeply appreciate the contribution of this thesis made in various ways by my friends and colleagues.

\section{References}

1. Neal MD, Sodhi CP, Dyer M, Craig BT, Good M, Jia H, Yazji I, Afrazi A, Richardson WM, Beer-Stolz D et al: A critical role for TLR4 induction of autophagy in the regulation of enterocyte migration and the 
pathogenesis of necrotizing enterocolitis. J Immunol 2013, 190(7):3541-3551.

2. Perrone EE, Jung E, Breed E, Dominguez JA, Liang Z, Clark AT, Dunne WM, Burd EM, Coopersmith CM: Mechanisms of methicillin-resistant Staphylococcus aureus pneumonia-induced intestinal epithelial apoptosis. Shock 2012, 38(1):68-75.

3. Vlug MS, Diepenhorst GM, van Koperen PJ, Renooij W, de Smet MB, Slors JF, Boermeester MA, Bemelman WA: Intestinal barrier function in patients undergoing colectomy. Colorectal disease : the official journal of the Association of Coloproctology of Great Britain and Ireland 2011, 13(12):14321437.

4. Nathan, J., Klingensmith, Craig, M., Coopersmith: The Gut as the Motor of Multiple Organ Dysfunction in Critical Illness. Critical Care Clinics 2016, 32(2):203-212.

5. Sartelli M: Evaluation and management of abdominal sepsis. Curr Opin Crit Care 2020, 26(2):205211.

6. The F, Cailotto C, van der Vliet J, de Jonge WJ, Bennink RJ, Buijs RM, Boeckxstaens GE: Central activation of the cholinergic anti-inflammatory pathway reduces surgical inflammation in experimental post-operative ileus. Br J Pharmacol 2011, 163(5):1007-1016.

7. Ghia JE, Blennerhassett P, Collins SM: Vagus nerve integrity and experimental colitis. American Journal of Physiology Gastrointestinal \& Liver Physiology 2007, 293(3):G560.

8. Borovikova LV, Ivanova S, Zhang M, Yang H, Botchkina GI, Watkins LR, Wang H, Abumrad N, Eaton JW, Tracey KJ: Vagus nerve stimulation attenuates the systemic inflammatory response to endotoxin. Nature 2000, 405(6785):458-462.

9. Vida G, Pena G, Deitch EA, Ulloa L: a7-Cholinergic Receptor Mediates Vagal Induction of Splenic Norepinephrine. Journal of Immunology 2011, 186(7):4340.

10. Zi SF, Li JH, Liu L, Deng C, Ao X, Chen DD, Wu SZ: Dexmedetomidine-mediated protection against septic liver injury depends on TLR4/MyD88/NF-KB signaling downregulation partly via cholinergic anti-inflammatory mechanisms. Int Immunopharmacol 2019, 76:105898.

11. Taniguchi T, Kurita A, Kobayashi K, Yamamoto K, Inaba H: Dose- and time-related effects of dexmedetomidine on mortality and inflammatory responses to endotoxin-induced shock in rats. $J$ Anesth 2008, 22(3):221-228.

12. Gu J, Chen J, Xia P, Tao G, Zhao H, Ma D: Dexmedetomidine attenuates remote lung injury induced by renal ischemia-reperfusion in mice. Acta Anaesthesiol Scand 2011, 55(10):1272-1278.

13. Hofer S, Steppan J, Wagner T, Funke B, Lichtenstern C, Martin E, Graf BM, Bierhaus A, Weigand MA: Central sympatholytics prolong survival in experimental sepsis. Crit Care 2009, 13(1):R11.

14. Kang SH, Kim YS, Hong TH, Chae MS, Cho ML, Her YM, Lee J: Effects of dexmedetomidine on inflammatory responses in patients undergoing laparoscopic cholecystectomy. Acta Anaesthesiol Scand 2013, 57(4):480-487.

15. Wang $Y, X u X$, Liu H, Ji F: Effects of dexmedetomidine on patients undergoing radical gastrectomy. J Surg Res 2015, 194(1):147-153. 
16. Tasdogan M, Memis D, Sut N, Yuksel M: Results of a pilot study on the effects of propofol and dexmedetomidine on inflammatory responses and intraabdominal pressure in severe sepsis. $J$ Clin Anesth 2009, 21(6):394-400.

17. Li R, Lai IK, Pan JZ, Zhang P, Maze M: Dexmedetomidine Exerts an Anti-inflammatory Effect via a2 Adrenoceptors to Prevent Lipopolysaccharide-induced Cognitive Decline in Mice. Anesthesiology 2020, 133(2):393-407.

18. Yeh YC, Wu CY, Cheng YJ, Liu CM, Hsiao JK, Chan WS, Wu ZG, Yu LC, Sun WZ: Effects of Dexmedetomidine on Intestinal Microcirculation and Intestinal Epithelial Barrier in Endotoxemic Rats. Anesthesiology 2016, 125(2):355-367.

19. Barr J, Fraser GL, Puntillo K, Ely EW, Gélinas C, Dasta JF, Davidson JE, Devlin JW, Kress JP, Joffe AM et al: Clinical practice guidelines for the management of pain, agitation, and delirium in adult patients in the intensive care unit. Critical care medicine 2013, 41(1):263-306.

20. Otani S, Coopersmith CM: Gut integrity in critical illness. Journal of intensive care 2019, 7:17.

21. Clark JA, Coopersmith CM: INTESTINAL CROSSTALK. Shock 2007, 28(4):384-393.

22. Mittal R, Coopersmith $\mathrm{CM}$ : Redefining the gut as the motor of critical illness. Trends in Molecular Medicine 2014, 20(4):214-223.

23. Ross JT, Matthay MA, Harris HW: Secondary peritonitis: principles of diagnosis and intervention. BMJ 2018, 361:k1407.

24. Singer M, Deutschman CS, Seymour CW, Shankar-Hari M, Annane D, Bauer M, Bellomo R, Bernard GR, Chiche JD, Coopersmith CM et al: The Third International Consensus Definitions for Sepsis and Septic Shock (Sepsis-3). JAMA 2016, 315(8):801-810.

25. Surviving Sepsis Campaign: International Guidelines for Management of Sepsis and Septic Shock: 2016. Intensive Care Medicine 2017, 43(3):304-377.

26. Sartelli M: Evaluation and management of abdominal sepsis. Current Opinion in Critical Care 2020, 26.

27. Chao R, Xiu-Hua L, Shi-Bin W, Li-Xue W, Ning D, Yao W, Yong-Ming Y: Activation of Central Alpha 7 Nicotinic Acetylcholine Receptor Reverses Suppressed Immune Function of T Lymphocytes and Protects Against Sepsis Lethality. International Journal of Biological Sciences 2018, 14(7):748.

28. van Maanen MA, Stoof SP, Larosa GJ, Vervoordeldonk MJ, Tak PP: Role of the cholinergic nervous system in rheumatoid arthritis: aggravation of arthritis in nicotinic acetylcholine receptor a7 subunit gene knockout mice. Annals of the rheumatic diseases 2010, 69(9):1717-1723.

29. Li DJ, Fu H, Tong J, Li YH, Qu LF, Wang P, Shen FM: Cholinergic anti-inflammatory pathway inhibits neointimal hyperplasia by suppressing inflammation and oxidative stress. Redox Biology 2018, 15:22-33.

30. Bencherif M, Lippiello PM, Lucas R, Marrero MB: Alpha7 nicotinic receptors as novel therapeutic targets for inflammation-based diseases. Cellular \& Molecular Life Sciences Cm/s 2011, 68(6):931949. 
31. Cedillo JL, Arnalich F, Martín-Sánchez C, Quesada A, Rios JJ, Maldifassi MC, Atienza G, Renart J, Fernández-Capitán $\mathrm{C}$, García-Rio $\mathrm{F}$ et al: Usefulness of a7 nicotinic receptor messenger RNA levels in peripheral blood mononuclear cells as a marker for cholinergic antiinflammatory pathway activity in septic patients: results of a pilot study. The Journal of infectious diseases 2015, 211(1):146-155.

32. Nestor JE: Dexmedetomidine for Long-Term Sedation Investigators. Dexmedetomidine vs Midazolam or Propofol for Sedation during Prolonged Mechanical Ventilation: Two Randomized Controlled Trials. American journal of respiratory and critical care medicine 2012, 186(11):1190-1190.

33. Li CJ, Wang BJ, Mu DL, Hu J, Guo C, Li XY, Ma D, Wang DX: Randomized clinical trial of intraoperative dexmedetomidine to prevent delirium in the elderly undergoing major non-cardiac surgery. The British journal of surgery 2020, 107(2):e123-e132.

34. Hu J, Zhu M, Gao Z, Zhao S, Feng X, Chen J, Zhang Y, Maze M: Dexmedetomidine for prevention of postoperative delirium in older adults undergoing oesophagectomy with total intravenous anaesthesia: A double-blind, randomised clinical trial. European journal of anaesthesiology 2020.

35. Xiang H, Hu B, Li Z, Li J: Dexmedetomidine controls systemic cytokine levels through the cholinergic anti-inflammatory pathway. Inflammation 2014, 37(5):1763-1770.

36. Bruewer M, Luegering A, Kucharzik T, Parkos CA, Madara JL, Hopkins AM, Nusrat A: Proinflammatory cytokines disrupt epithelial barrier function by apoptosis-independent mechanisms. J Immunol 2003, 171(11):6164-6172.

37. Yoseph BP, Klingensmith NJ, Liang Z, Breed ER, Burd EM, Mittal R, Dominguez JA, Petrie B, Ford ML, Coopersmith CM: Mechanisms of Intestinal Barrier Dysfunction in Sepsis. Shock 2016, 46(1):52-59.

38. Murray MJ, Barbose JJ, Cobb CF: Serum D(-)-lactate levels as a predictor of acute intestinal ischemia in a rat model. Journal of Surgical Research 1993, 54(5):507-509.

\section{Figures}




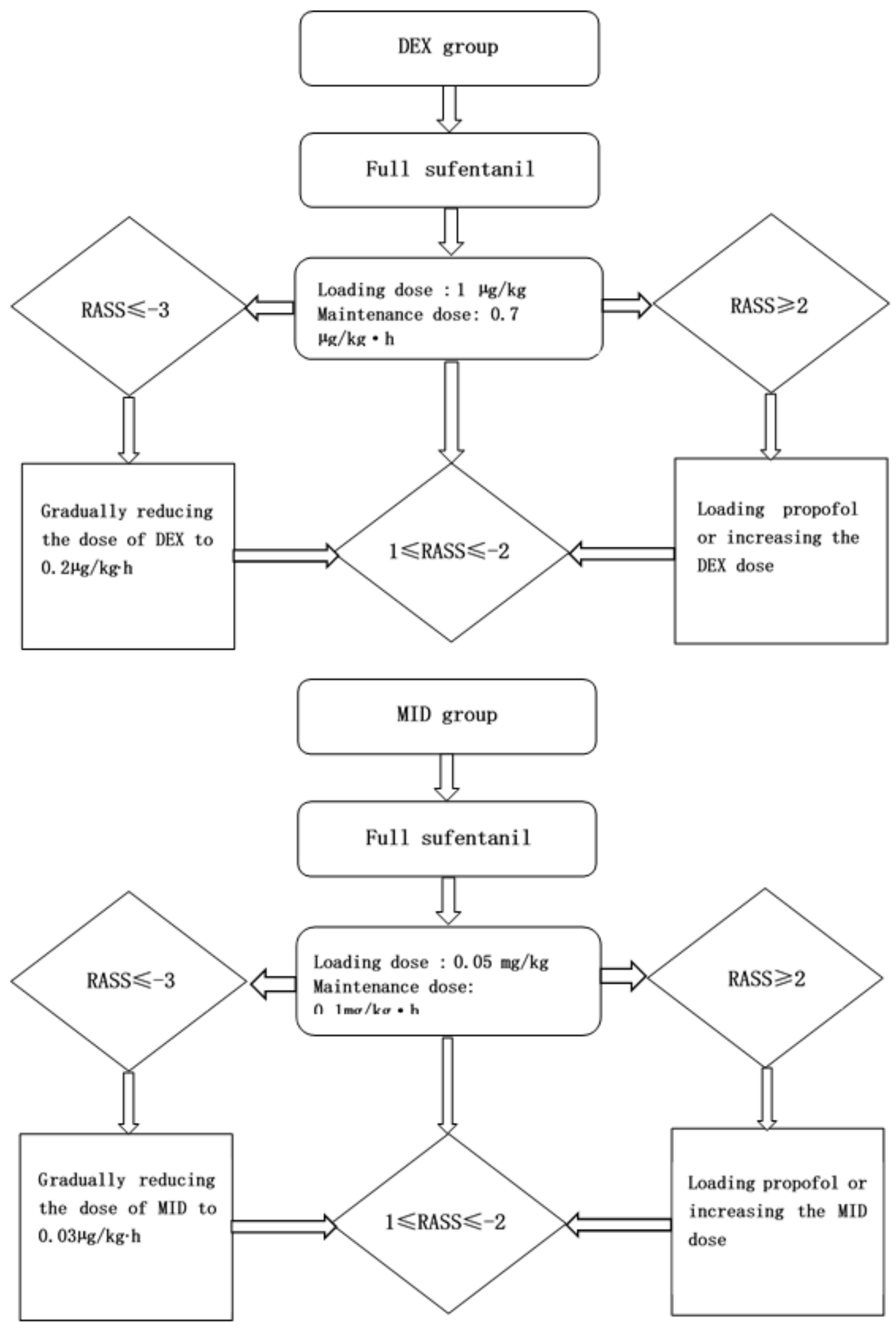

\section{Figure 1}

Sedative flow of the DEX and MID groups 


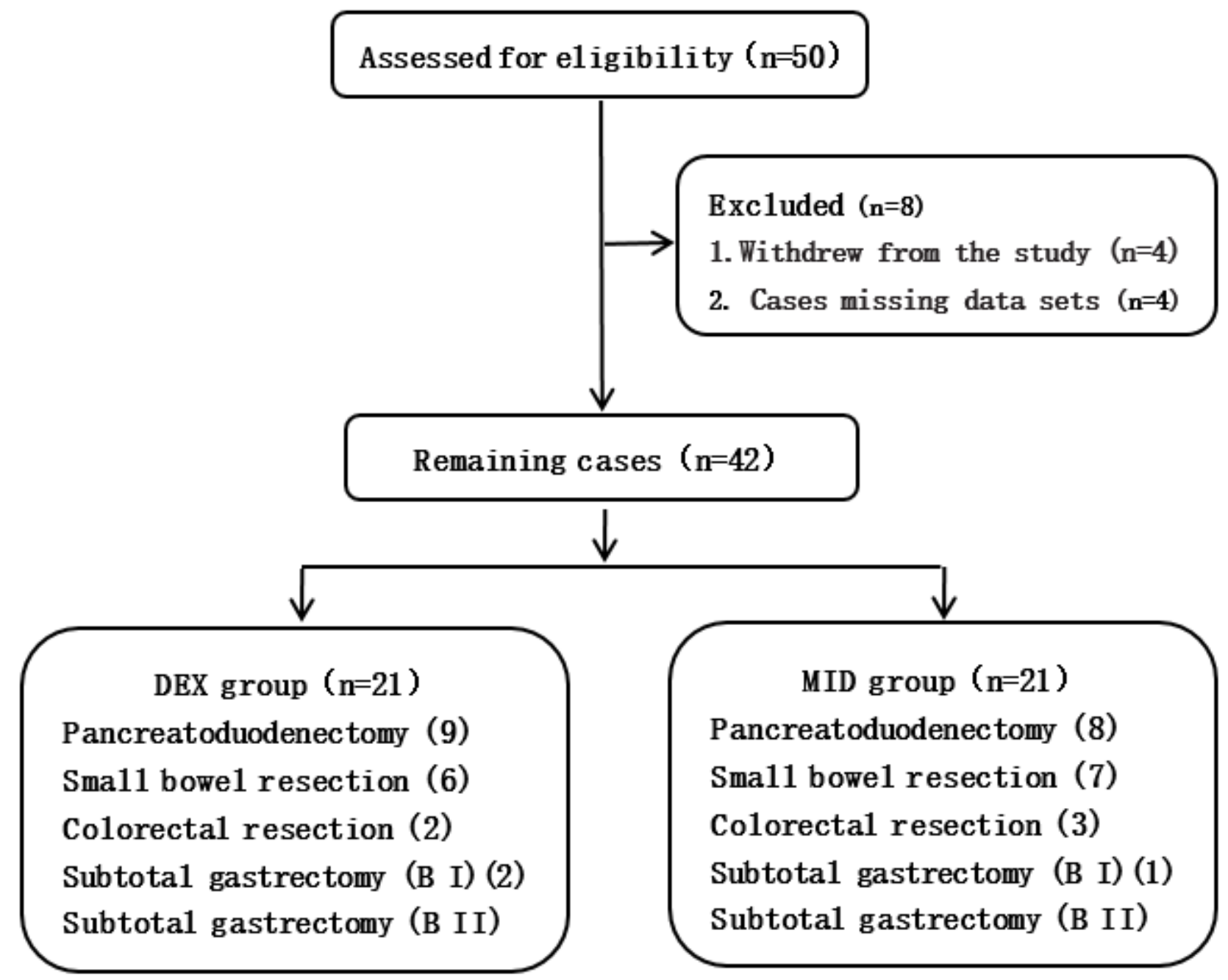

Figure 2

Flow chart of patient inclusion 